\title{
VIABILITY OF NEWLY DEVELOPED PROCESS FOR CATALYTIC CONVERSION OF BIO-GLICERIN INTO VARIOUS "GREEN CHEMICAL"AND DEVELOPMENT OPPORTUNITIES FOR SERBIA
}

\author{
Zoran Popović* \\ Institute for Chemistry, Technology and Metallurgy, University of Belgrade, Serbia \\ Ivana Souček \\ University of Chemistry and Technology (UCT), Prague, Czech Republic \\ Dalibor Marinković \\ Institute for Chemistry, Technology and Metallurgy, University of Belgrade, Serbia \\ Ozren Ocić \\ Institute for Chemistry, Technology and Metallurgy, University of Belgrade, Serbia
}

Crude bio-glycerin is a by-product in the production of biodiesel. An economically efficient valorization of crude bio-glycerin usually significantly defines the profitability of the biodiesel plant. A strong technological development in recent years has provided an additional option for economically viable use of glycerin through conversion of a crude bio-glycerin, in first step into a technical glycerin, and then further by use of new catalytic processes into various "green chemicals", such as epichlorohydrin, propylene-glycol, bio-methanol, acrolein, acrylic acid, etc. Many of those newly-developed technologies are already in use on an industrial scale. This trend had caused a growth in demand of bio-glycerin, which gradually has led to a rise of bio-glycerin's market value and contributed to an overall growth in profitability of biodiesel industry. This paper has the aim to assess: global availability of the emerging technologies intended for valorization of bio-glycerin via production of commercially attractive "green chemicals", local and regional availability of bio-glycerin for an economically justifiable production of "green chemicals", and local and regional potential for marketing of the most viable "green chemicals". All market assessments and expected challenges that are stated in this paper took into account a reality that development of the biodiesel industry is still largely influenced by government subsidies and mandates, and also by a price of crude oil.

Key words: Biodiesel Industry, Crude Bio-Glycerin Market, Bio-Glycerin to „Green Chemicals“, Serbia's Development Opportunities

\section{INTRODUCTION}

Conventional biodiesel (Methyl Esters of Fatty Acids or abbreviated FAME) is manufactured by trans-esterification of vegetable oils and/or animal fats by methanol in presence of catalyst.

By-product (or co-product) of transesterification process is crude bio-glycerin. For every $1,000 \mathrm{~kg}$ of FAME manufactured by transesterification, approximately $100 \mathrm{~kg}$ of crude bio-glycerin is also produced. An economically efficient valorization of crude bio-glycerin lowers the cost of FAME production and usually significantly defines the profitability of FAME manufacturing. European biodiesel industry has already gone through a period of crisis from 2008 to 2010 , when extremely rapid growth in FAME production caused enormous oversupply of bio-glycerin, so that glycerin had got minor or no economic value.

Almost an immediate response to this situation had been a strong technological development, and through which the invention of a whole range of new catalyzed thermochemical processes for converting crude bio-glycerin into profitable chemical intermediates. Many of those processes have already been translated to the level of commercial exploitation on an industrial scale (epichlorohydrin, propylene-glycol, bio-methanol, acrolein, acrylic acid, ...) [18]. This trend had caused a growth in demand of bio-glycerin, which gradually has led to a rise of bio-glycerin's mar- 
ket value and contributed to an overall growth in profitability of biodiesel industry.

The volume of biodiesel production in Serbia is currently at a very low level. However, there are at least a doezen factories that currently produce biodiesel on an industrial scale in the neighboring countries (Hungary, Romania, Bulgaria and Croatia) [06].

In this sense, this paper has the aim to assess:

1) global availability of the emerging technologies intended for valorization of bio-glycerin via production of commercially attractive chemical derivatives,

2) local and regional availability of bio-glycerin for an economically justifiable production of chemical derivatives that are identified under item (1), and

3) local and regional potential for marketing of products that are identified under item (1).

All market assessments and expected challenges that are stated in this paper took into account a reality that development of the biodiesel industry is still largely influenced by government subsidies and mandates, and also by a price of crude oil [16].

\section{GLYCERIN MARKET WORLDWIDE}

Glycerin (glycerol or 1,2,3-propanetriol) is the oldest organic molecule isolated by human beings in a such a way that fats were heated in the presence of ash in order to produce soap, and that was happened around $2800 \mathrm{BC}$. In the modern era, the first industrial application of glycerin was by the military industry in the production of nitroglycerin as a strategically important raw material for the production of explosives and gunpowder (1840's). The next industrial applications were use of technical glycerin as raw material in the manufacture of alkyd resins for coatings industry and various direct uses of high-quality glycerin in the formulations of personal care products and pharmaceuticals (1920's).

Due to the rapid growth in demand of glycerin and its limited supply from production of soaps and oleochemicals, as of mid-1930s began production of petrochemical (synthetic) glycerin. From this moment, glycerin had been an extremely profitable chemical product, and highpurity grades of glycerin were sold even at prices ranging from 2,500 to 3,500 USD per $t$ [02].

Since 2003, however, the boom in FAME pro- duction and rapidly increasing glycerin oversupply caused a dramatic fall in the price of both refined and crude glycerin. Very soon after that, the production of synthetic glycerin worldwide was almost completely abandoned. Prices had continued to drop and in 2009-2010 the European price of highly-purified glycerin was less than 320 EUR per $t$, while the prices of crude bio-glycerin ranged from 80-100 EUR per $t$ (for GMO-free origin) to zero (for GMO origin). Crude bio-glycerin of lower quality had become a waste material of no economic value, except the incinerated as a low-calorific fuel [11]. Changes in average structure of the origin of glycerin at global level are shown in Figure 1. As can be seen, an increased contribution of biodiesel is obvious, from $9 \%$ in 2000 to $64 \%$ in 2013 . Changes in Europe were even more radical, because in 2014 the share of FAME in structure of glycerin produced reached over $85 \%$ [04]. Global biodiesel production has grown from about $200,000 \mathrm{t}$ in 2003 to about 29 million t produced in 2014 . Practically, this means that in just 11 years global supply of glycerin from biodiesel production (i.e. bio-glycerin) increased about 150 times. The worldwide leader in consumption and production of glycerin is Europe. With current consumption of around 1 million t per year (toy) its share in global demand is $28-30 \%$, while the share in global production is even higher, approximately $33 \%$. The major end-uses of glycerin in Europe are production of toiletries \& cosmetics, food \& beverages industry, production of polyether polyols and pharmaceutical industry. Besides Europe very large users of glycerin are China $(27 \%)$ and the United States (21\%). Cumulative share of all other countries in global consumption of glycerin is less than $25 \%$.

Today, glycerin is among the most important industrial oleochemicals, along with fatty acids, fatty alcohols and FAME, and it has many end-uses since a variety of these applications is growing continuously.

Among these new applications are the newlydeveloped catalytic processes for conversion of refined glycerin into chemical intermediates.

Other new uses of glycerin with high market potential include replacement of more expensive sorbitol and propylene-glycol as sweeteners and humectant, as well as substitution of mono-ethylene-glycol with technical grade glycerin in formulations of windshield wash fluids and coolants and antifreeze products. The latest application of 
this kind is liquid for e-cigarettes which contain glycerin of Kosher quality (100\% GMO-free) in a function of the artificial sweetener. Thanks to the combination of new applications and mar- ket expansion in traditional markets, the price of glycerol partly recovered since the 2009-2010 historic lows (see Figure 2).

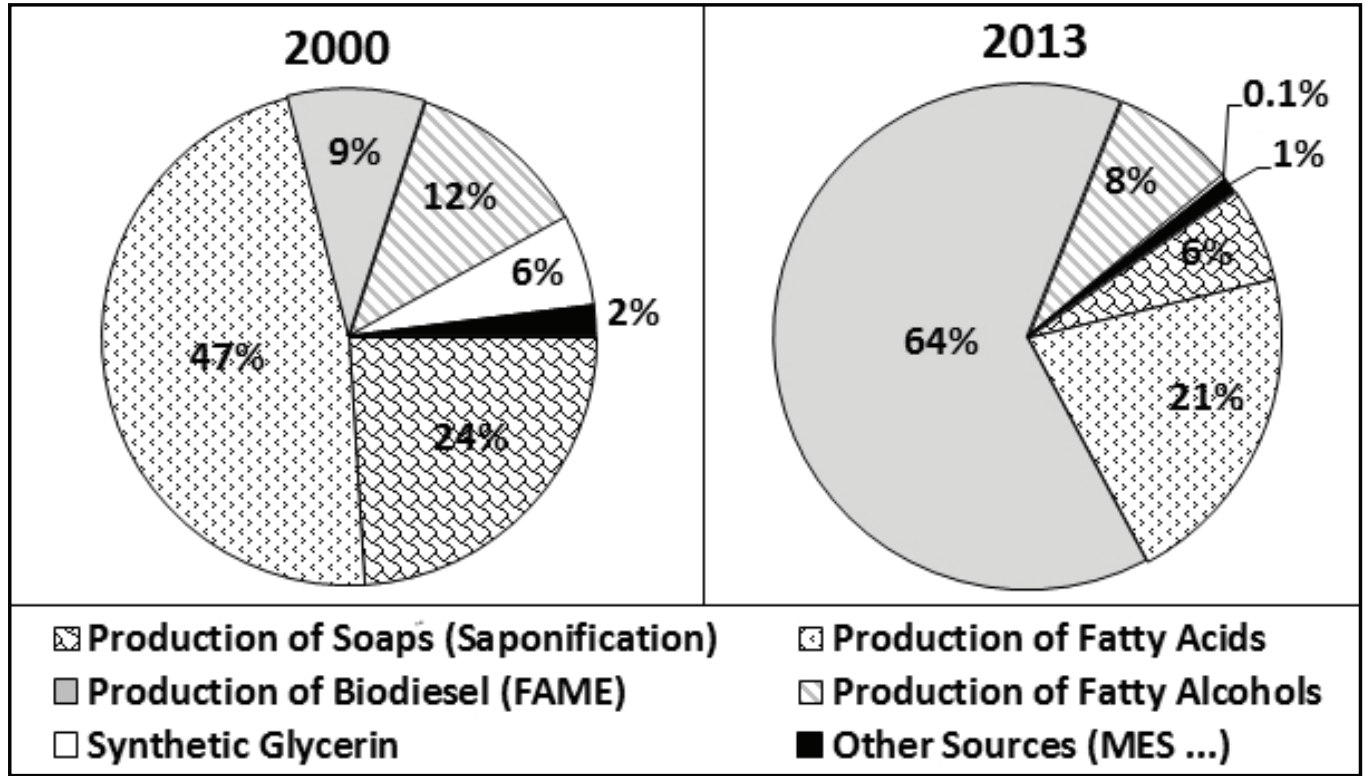

Figure 1: Glycerin in 21 ${ }^{\text {st }}$ century: Radical change of supply sources [04]

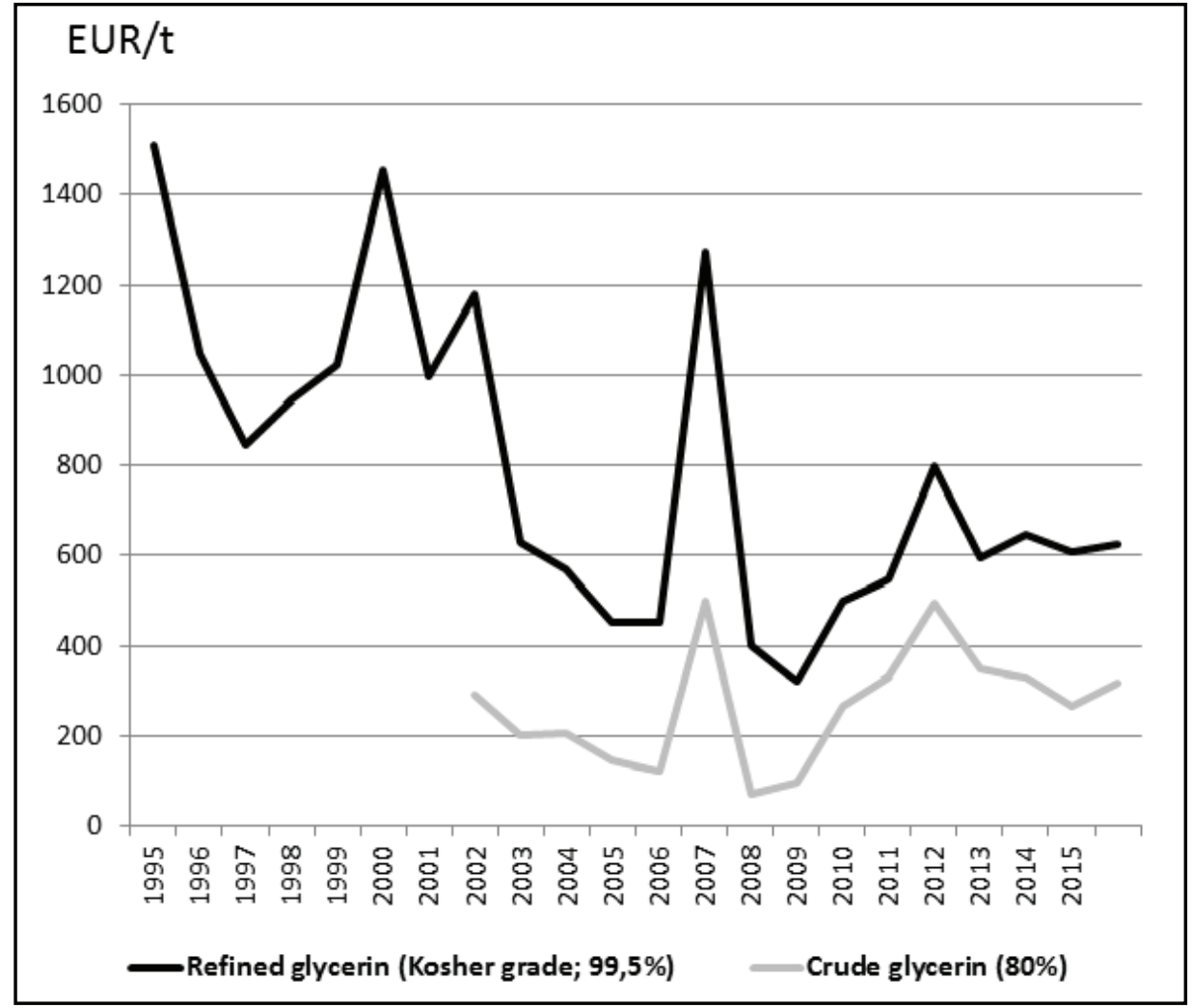

Figure 2: European spot prices of glycerin (CFR Rotterdam) [16]

Most recent prognostic studies predict growth in global consumption of glycerin up to 2020 by very high compound annual growth rates (CAGRs), ranging from 6.3 to $6.8 \%[08,01]$. The largest sector demand for refined glycerin will remain the production of pharmaceuticals and toiletries, but uses of highly purified glycerin in the food industry \& soft drinks industry will grow extremely 
fast, at CAGR of about $8.5 \%$. However, the highest growth at CAGR of over $10 \%$ will record uses of refined glycerin in catalytic processes to produce chemical intermediates.

\section{GLYCERIN MARKET IN SERBIA AND NEIGBORING COUNTRIES}

Today, Serbia has at its disposal a certain amount of crude bio-glycerin from FAME production, but in 2014 this quantity was not greater than $300 \mathrm{t}$. Production of refined glycerin does not exist, and the needs of the domestic market for glycerin are covered from imports. In recent years, annual imports of refined glycerin ranged from 2,000 to $2,100 \mathrm{t}$, whereas import of crude glycerin was negligible. Major domestic consumers of refined glycerin are production of nitroglycerin, tobacco industry as well as manufacturers of soaps, toiletries and cleansers.

By adoption of (i) Ministerial Council Decision 2012/04/MC-EnC within the Energy Community (EC) in October 2012, and (ii) National Renewable Energy Action Plan in June 2013, the Republic of Serbia took over obligations arising from the provisions of the Renewable Energy Directive (RED) 2009/28/EC with regard to achieving the percentages that are targeted in 2020 for the overall share of energy from renewable sources and for the share of energy from renewable sources in transport. The bulk of mandates within EC member states continue to come from the EU-27, where the RED Directive specified a $10 \%$ renewable energy content in sector of road transport by 2020 [17].

Serbia has an obligation to reach by 2020 some $27 \%$ of energy consumption from renewable sources. It is uncertain how Serbia will meet this target and increase share of renewable energy in total energy consumption for over $5 \%$ in only five years.

Each EC member state has the freedom to decide how they will achieve that target through rise of energy from renewable sources in electrical energy generation, in the sector of heating and cooling, as well as in the transport sector. Above mentioned the National Renewable Energy Action Plan includes the projection that by 2020 the share of renewables in the transport sector will reach mandatory level of $10 \%$. Having in mind projections of domestic consumption of energy in sector of transport, that means that domestic consumption of renewables might reach in 2020 a level of over 135,000 to $245,000 t$ of oil equivalent (toe) [10]. Most of this renewables will be biofuels.

Targets of Serbia for energy from renewable sources in transport sector refer to the "consumption“, and not for the „production“. On us is whether the targeted "consumption" we want to achieve through use of renewables of domestic origin or by import.

Orientation of Serbia on imports of biofuels automatically means the reduction of market for domestic suppliers of motor fuels (at level that corresponded to the quantity of imported biofuel intended for blending). For JSC "NIS", who is manufacturer, wholesaler and exporter of petroleum products, and also the owner of a chain of gas stations in Serbia and neighboring countries, this situation also means the reduction of its market potential for placement of motor fuels in the region.

Therefore, from an economic point of view, it is quite realistic to expect the re-start of domestic production of biofuels in existing plants. However, it is important to underline that domestic production of biofuels starts from an almost negligible level, but the capacities for production of around 100,000 tpy were constructed but they are not in operation.

On the other hand, there are two "weak points" related to the development of biofuel production in Serbia.

First is the fact that according to all reliable forecasts, the producer prices of conventional fuels in the next 5-6 years will not exceed a level that allows the competitiveness of biofuels. It would be unrealistic to count with high-level production and sale of FAME in Serbia if its price is 0.62-0.64 EUR per liter [01], while you can buy petroleum diesel at price of 0.49-0.52 EUR per liter [05] .

Second, the biofuel industry is generally very dependent on legal regulations and incentives. In the past few years much has been done in Serbia on the first assignment, but up to now practically nothing happened on the implementation of this latter assignment.

Serbia already has the plants of industrial scale for the production of FAME, three of which are facilities of smaller size in operation with total capacity of around 6,500 tpy. There are also two larger FAME plants, one of which is idled $(100,000$ tpy located in Šid), and the other one which is still not completed $(35,000$ tpy located 
in Kruševac) [12]. Besides, over 1,000 individual farmers throughout Serbia are producing FAME for their own use in small on-farm production systems.

On the other hand, Serbia still has no facilities for the production of bioethanol or any of the advanced biofuels [12]. Moreover, there are no indications that such kind of facility could be built in Serbia in the near future. Therefore, it is highly probable that fulfillment of national targets on use of bio-renewables in transportation sector up to 2020 will be almost exclusively realized through the development of production and consumption of biodiesel consumption (production).
Bearing in mind benchmarks regarding use of renewables in transport sector which were imposed by the European Commission, then the expected development of "road“ diesel fuel consumption (production) in Serbia [13] and marginal percentages of biodiesel blending [10] the domestic needs for biodiesel in 2020 will be at a level of $113,000-179,000 \mathrm{t}$, and in 2025 at a level of 183,000-203,000 $t$ [10].

In addition, the significant amounts of bio-glycerin are already available in neighboring countries. Table 1 shows current quantities of crude bioglycerin that might be considered as available at regional level.

Table 1: Availability of bio-glycerin in neighboring countries (in tpy)

\begin{tabular}{|l|c|c|c|c|}
\hline \multicolumn{1}{|c|}{ Country } & $\begin{array}{c}\text { FAME capacity } \\
(2014)\end{array}$ & $\begin{array}{c}\text { FAME production } \\
(2014)\end{array}$ & $\begin{array}{c}\text { Bio-glycerinavailability* } \\
(2014)\end{array}$ & $\begin{array}{c}\text { Glycerin exports } \\
(2012-2014)\end{array}$ \\
\hline Romania & 277,000 & 160,000 & 16,000 & $15,035(2014)$ \\
\hline Bulgaria & 408,000 & $40,000(2013)$ & $4,000(2013)$ & $1,000-1,500$ \\
\hline Hungary & 162,000 & 190,000 & 19,000 & $17,650-19,300$ \\
\hline Croatia & 62,000 & 35,000 & 3,500 & $10,460(2014)$ \\
\hline FYR Macedonia & 20,000 & 6,500 & 650 & $630(2014)$ \\
\hline Greece & 812,000 & $140,000(2013)$ & $14,000(2013)$ & $16,835(2014)$ \\
\hline T O T A L: & $\mathbf{1 , 7 4 1 , 0 0 0}$ & $\mathbf{5 7 1 , 5 0 0}$ & $\mathbf{5 7 , 1 5 0}$ & $\mathbf{6 1 , 6 1 0 - 6 3 , 7 6 0}$ \\
\hline
\end{tabular}

*Based on fact that FAME production generates about $10 \%(\mathrm{w} / \mathrm{w})$ of bio-glycerin

Remark: Other neighboring countries currently do not have production of FAME.

The Croatian exports obviously include some re-export. Data on Bulgarian exports in 2014 are not available.

\section{AVAILABILITY OF CRUDE BIO-GLYCERIN IS EQUATION INVOLVING SEVERAL UNKNOWNS}

Global biodiesel industry consists today from facilities to produce FAME and so-called "renewable diesel" (which is also known as Hydrotreated Vegetable Oil or abbreviated HVO).

The first manufacturing plant to produce HVO was started on December 2007 in Finland by the petroleum company „Neste Oil“. HVO is produced by the hydrotreating vegetable oils and/or animal fats. The process of HVO manufacturing does not yield bio-glycerin, but other by-products such as naphtha (for petrochemical industry), LPG and propane. HVO has emerged as an attractive alternative to FAME in recent years. With this process the same kinds of vegetable oil feedstock that are used in FAME production are reacted with hydrogen in a process called hydrotreating - a common process in the pe- troleum industry. The primary advantage of HVO over FAME is that the product is chemically equivalent to petroleum diesel, so it can be used in diesel engines in higher concentration with no modifications (usually up -to $30 \%$ ). A disadvantage is higher capital costs of hydrotreating equipment when compared with equipment required to produce biodiesel, and thus HVO production requires larger scale to be economical. Today in Europe manufacturing of HVO have the Netherlands, Finland, Italy, Sweden, Germany, Spain and France. World production of biodiesel has grown throughout the entire first decade of the $21^{\text {st }}$ century. Globally, increase of biodiesel production has been realized in production by both FAME and HVO, although from 2010 this increase can mainly be attributed to rising HVO production. This trend is even more evident In Europe, where production of FAME experienced "peak" back in 2010, and since than the production of biodiesel (FAME + HVO) is maintained on an ongoing level only due to increased HVO production. 
Table 2: Production of biodiesel (FAME/HVO) in 2014 (in million t)

\begin{tabular}{|c|c|c|c|c|c|c|}
\hline & \multicolumn{2}{|c|}{ FAME } & \multicolumn{2}{c||}{ HVO } & \multicolumn{2}{c|}{ Total Biodiesel } \\
\hline & 2010 & 2014 & 2010 & 2014 & 2010 & 2014 \\
\hline World & 17.25 & 22.00 & 0.15 & 2.70 & 17.40 & 24.70 \\
\hline EU-28 & 9.35 & 8.10 & 0.15 & 1.45 & 9.50 & 9.55 \\
\hline \hline
\end{tabular}

The global biofuel industry is nowadays under great pressure. Crude oil and petroleum products prices collapsed towards the end of 2014 and into 2015, which very soon caused a huge enlarging of the margins between prices of biofuels and petroleum products. Slowly but surely such circumstances have reduced blending of biodiesel worldwide. Many European plants to produce FAME are either mothballed or running at low capacities due to a lack of demand. Although energy legislation still pushes the market participants to fulfill national mandates on blending biofuels, and although European Commission responded promptly and increased biodiesel premiums, some of market participants decided rather to pay penalties than to blend uncompetitive FAME.

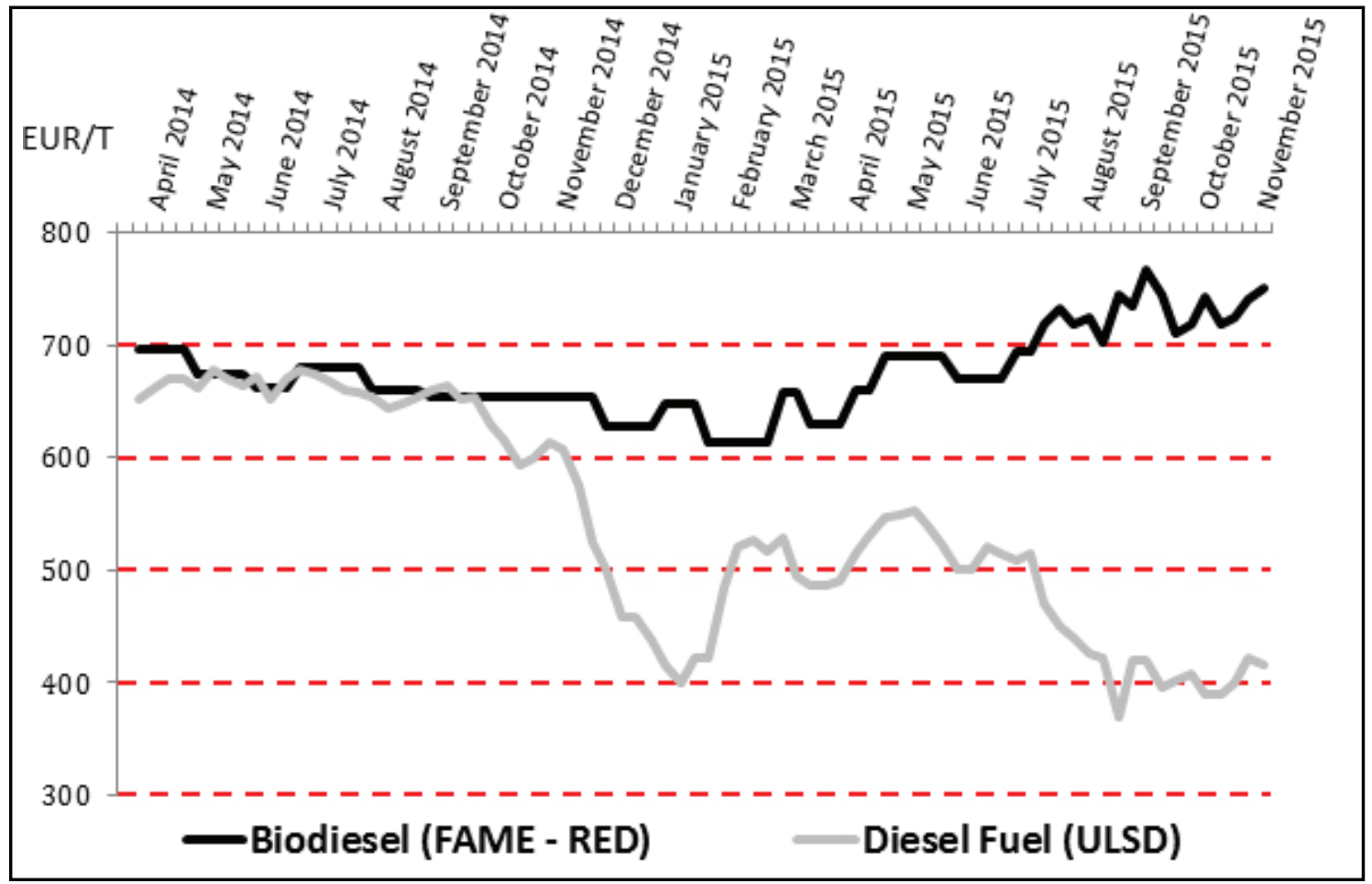

Figure 3: Historic prices of biodiesel (FAME - RED) and petroleum diesel fuel (ULSD) (spot prices FOB Rotterdam) [01, 14]

It should be underlined that problems of biodiesel industry have caused also a certain drop in global prices of the crude vegetable oils, and particularly drop in prices of waste and residual fats and oils, what is logical since the biodiesel industry is a major consuming sector of these feedstock . However, those price reductions were not sufficient to make biodiesel competitive in the motor fuel market. At the beginning of November 2015, an average EU's wholesale price for biodiesel net of duties and taxes stood at around 750 EUR per $t$, thus exceeding the price for off-road diesel fuel (diesel fuel which is not subject to duties and excise taxes that are applied to the retail sales of road-diesel fuel) by nearly 340 EUR per $t$.

Also, there are two new events that will mostly probably affect the future availability of bio-glycerin in Europe:

- In April 2015 by the European Parliament approved Directive 2015/1513/EU that says that first-generation biofuels (i.e. biofuels produced from food crops, which includes FAME) should not exceed $7 \%$ of the final energy consumption in transport by 2020 (less 
than $10 \%$ stated in RED Directive).

- Currently, a major turnaround is in progress in interregional trade of motor fuels. All analyzes indicate that in 2015 the United States will become a net exporter of motor gasoline, for the first time after many decades. This event will significantly change an established image according to which the European refineries marketed their own surpluses of gasoline fuels in the United States. It is likely that such kind of shift will have an impact on relations in European demand of motor fuels in favor of gasoline (and at the expense of diesel pool of refinery streams which also includes the biodiesel).

Respecting all the above stated, it could be concluded that availability of bio-glycerin has reached a historic „peak" at both worldwide level (most probably) and in Europe (definitely). Further, rise of glycerin availability will only be realized from increased production of certain oleochemicals (fatty acids, fatty alcohols and methyl ester sulfonates). However, having in mind the current share of these productions in overall global supplies of glycerin, and the fact that new production capacity of these oleochemicals is built almost exclusively in the Asia-Pacific region, it seems that a market participant who intends to consume glycerin as feedstock in the manufacturing plant which is located in Europe, should not in future count to enlarged level of glycerin availability.

\section{PROBLEMS IN VALORIZATION OF CRUDE BIO-GLYCERIN WITHIN CHEMICAL INDUSTRY}

The first and essential factor in assessing the technoeconomic viability to valorize crude bioglycerin through its use in chemical syntheses is its origin, i.e. type of processes to produce biodiesel which yields it. This refers to conventional process using a homogeneous catalyst or a process using a heterogeneous catalyst on carrier.

Homogenous catalysts are alkaline metal hydroxides and alkoxides as well as sodium or potassium carbonates. Since these catalysts react with components of vegetable oils and fats, and methanol, this kind of production process yields crude bio-glycerin in mixture with a lot of impurities. Advantages of that production process are high yield of FAME and cheap catalysts.

Heterogeneous catalysts are systems of alkaline or alkaline -earth metal or their oxides on carriers. Main advantage of heterogeneous catalyst is that it can be recycled and re-used several times with easier separation of the final reaction product. Heterogeneous catalysis in biodiesel production, unlike homogeneous catalysis, removes the costly and time-consuming water washing and neutralization steps to separate and recover the spent catalyst. Also, contaminated water from homogeneous catalysis process is greatly reduced and the need for waste water treatment minimized.

The problems for the use of crude bio-glycerin in chemical syntheses and other industrial applications are its high contamination with toxic methanol, high content of organic salts and fee fatty acids (FFAs), as well as its substantial color (from yellow to dark brown). A crude bio-glycerin produced from biodiesel process using heterogeneous catalysts is somewhat cleaner comparing to a crude bio-glycerin produced by conventional homogenous biodiesel processes. However, it should be bare in mind that all existing operating and preserved facilities for biodiesel production in Serbia use conventional process.

Glycerol fraction formed in the process of biodiesel production generally contains 23 to $63 \mathrm{wt}$ \% of crude bio-glycerin and more than $10 \mathrm{wt}$ \% of methanol. The rest is mixture of water, methyl esters and lipids, inorganic salts (catalyst residues), FFAs, unreacted mono-, di-, and tri-glycerides, and a variety of non-glycerin organic compounds in small percentages. These impurities make the crude bio-glycerin obtained in the process of decanting mixtures of biodiesel plus crude bio-glycerin unsuitable for direct application in the most traditional uses of glycerin.

To convert crude bio-glycerin into the product of commercial value is necessary to conduct the operations of removing water, methanol and its salts, and undissolved impurities (there are 5-7 wt. \% of insoluble salts in crude bio-glycerin from process of transesterification with homogeneous alkali catalyst). Or in other words, it is necessary to carry out the process of refining (purification) which is realized in two steps:

1) Getting demethylated bio-glycerin=Flash evaporation+Acidulation+Filtration ( eventually with the addition of auxiliary chemical agents)

2) Preparation of refined glycerin=Membrane filtration+Reverse osmosis+Drying+Disti 
llation. The process of flash evaporation, besides elimination of water, also removes most of the methanol, which is an easy-volatile organics. Acidulation includes treatment with $\mathrm{HCl}$ or $\mathrm{H} 2 \mathrm{SO} 4$ to neutralize the traces of the catalyst and causes hydrolysis of the soaps present in the crude bio-glycerin. That leads to the formation of insoluble salts, which are then removed (partially by decantation, and completely by filtration). Filtration with the addition of chemical agent that react with impurities gives de-methylated bio-glycerin with glycerin content of 80-90 wt. \%.

The minimum conditions for bio-glycerin to have any commercial value are glycerol content over 80 wt. \%, and methanol content below 1 wt. \%. In fact, bio-glycerin which does not fulfill these conditions can be used only as an energy source (incineration for heat production, fuel for steam boilers, and agent for improving the combustion properties of biogas in anaerobic digesters), then as an additive for certain types of cattle fodder (improves the ruminant's feed to weight gain ratio), and from recently as an auxilliary agent for cements of enhanced performance (replacing petrochemical amines and glycols).

Further purification of demethylated bio-glycerin leads to obtaining refined glycerin:

- Technical grade glycerin, 95.5 to $98.0 \%$ purity, suitable for production of chemicals and use in many other industrial applications, with exception of uses in food industry, pharmaceutical industry and industry of toiletries and cosmetics;

- Pharmaceutical grade glycerin (USP, Ph. Eur.), $99.5 \%$ and $99.7 \%$ purity, derived from vegetable oils and animal fats, suitable for food products, pharmaceuticals, toiletries and cosmetics;

- Kosher/Halal grade glycerin, high-purity, derived $100 \%$ from vegetable oils suitable for use in Kosher and Halal foods.

Treatment to reach Pharmaceutical or Kosher/ Halal glycerin through process of purification when it starts from crude bio-glycerin is complex and it requires serious CAPEX and OPEX, primarily those in distillation section.

Crude glycerin which is a by-product in production of fatty acids and fatty alcohols ("splitter crude glycerin") is not contaminated with methanol and catalyst's salts, and also has a much lower content of other organic impurities. This glycerin is usually completely converted into refined products, and mostly to those of Pharmaceutical or Kosher/Halal quality. CAPEX and OPEX correlated to process of "splitter crude glycerin" purification are significantly lower than those in treatment of crude bio-glycerin.

Crude bio-glycerin, even with the content of glycerin over $80 \%$, cannot be treated in a traditional oleochemical refinery for purification of the "splitter crude glycerin", since it could damage the equipment and pipelines. It must be treated in the purposely-built refinery where purification usually goes up to an improved technical quality (> 97 wt. \%).

The conversion of crude bio-glycerin into technical glycerin provides to owner of biodiesel factory certain advantages through eliminating the high costs of the toxic waste treatment and increasing the value of the products intended for market. Of course, there is a possibility of further purifying up to the Pharmaceutical or Kosher/Halal quality, but economic viability of this technological operation is highly problematic - it exists only within largescale biodiesel factories where the profitability is expected to benefit from a high integration of all material and energy streams and lowered CAPEX and OPEX per unit of final products.

Crude bio-glycerin from FAME plant based on esterification of waste fats or used cooking oil (UCO) is glycerin of worst quality in terms of its further conversion to the refined grades. Due to the high content of saturated fatty acids, this crude bio-glycerin has a high melting point, high viscosity and often even contains solid particles. An analysis from 2011, aimed to estimate the cost of crude bio-glycerin purification up to 98 wt. $\%$ (by combination of evaporation, acidulation, filtration/centrifugation, and column distillation), calculated the lowest OPEX for glycerin purification at level of 0.15 USD per kg [04]. Measured in current prices, it is about 0.16 USD per kg.

High investment expenditures (CAPEX) effectively minimize the economic viability of construction of process units for glycerin refining within small and medium biodiesel (FAME) factories. It is estimated that, in general, there is no economic viability for construction of glycerin refinery within biodiesel factory with a capacity < 10,000 tpy.

Smaller FAME producers, however, may find an economic interest in agglomerating all their 
own available quantities of crude bio-glycerin in a single location to enable an economically sized refinery for purification feed glycerin up to the products of technical quality. This refined glycerin would found applications as raw-material in the traditional and newly-developed syntheses of organic chemical industry.

\section{APPLICATIONS OF BIO-GLYCERIN IN CATALYTIC SYNTHESIS TO PRODUCE VARIOUS VALUE ADDED ORGANIC CHEMICALS}

There are two approaches for conversion of crude bio-glycerin into value-added organic chemicals, and substitution of deficient or expensive petrochemicals: (a) Biotechnological conversion, and (b) Catalytic conversion. This paper discussed only catalyzed conversions, since biotechnological conversions of bio-glycerin into organic chemicals still have not been transferred into industrial practice, according to the authors' best knowledge.

Catalytic conversions of bio-glycerin can be further divided into traditional and newly-developed processes.

\section{Traditional processes}

Traditional catalytic syntheses include several chemicals, but of more serious commercial value are only nitroglycerin (as component of almost all explosives and smokeless gunpowder as well as cardiovascular or therapeutic agent for the treatment of bronchial asthma in medicine) and glycerol esters (a commercially the most important is glycerol-triacetate). Today, productions of nitroglycerin and glycerol esters participate in global consumption of glycerin with $1.5 \%$ and $6 \%$, respectively.

The market analysis of Serbia and the neighboring countries is made, which confirmed sufficient regional demand to absorb the entire output of nitroglycerin or glycerol-triacetate from an industrial facility of economically reasonable size. The production of nitroglycerin was established long time ago within company "MB-Namenska" at location near town of Lucani. This same company had also a facility to produce glycerol-triacetate (it is generally known by trade name Triacetin $®$ ), but it was destroyed during NATO bombing campaign in 1999.

\section{Glycerol-triacetate (Triacetin $\left.{ }^{\circledR}\right)$}

Triacetin $®$ has many usages, but over one-third of demand can be attributed to tobacco industry where it is used as a binder for cellulose acetate tows that bundle forms a cigarette filter. Other more important applications include its use as binder for foundry sands, gelling agent for gunpowder and explosives, and plasticizer (for cellulosic materials, printing inks, textile dyes, adhesives etc.) [03]. Food grade Triacetin ${ }^{\circledR}$ is used as an auxiliary agent (humectant, plasticizer or solvent) in formulations of various pharmaceuticals and cosmetics (for instance, Triacetin ${ }^{\circledR}$ is irreplaceable component of several erectile-dysfunction drugs, such as "Viagra" and "Cialis") and as food additive (known by code E-1518 it is primarily used as a humectant). The most recent application of this commercially most important glycerol ester is its role in partial replacement of the health-risky phthalates within PVC industry [09].

Global market for Triacetin $®$ is around 150,000 tpy. The estimated demand and production in Europe (excluding Russia) are around 37,000 and 31,000 tpy, respectively [19].

There is not a single manufacturer of Triacetin $₫$ in Central, Eastern and South-Eastern Europe. Serbia imports about 200 tpy for the needs of local tobacco factories, plus $\sim 50$ tpy for other uses. Only seven neighboring countries, plus Greece, import additional 2,800 tpy .

Global demand of Triacetin $\AA$ will continue to grow by CAGR ranging from $6 \%$ to $8 \%$, which could be an expected growth for a product of "green chemistry", but is also an extraordinarily dynamic growth for one traditional organic compound.

The current prices of technical grade Triacetin $₫$ (98 wt. \%) range from 1,200 to 1,300 EUR per t. Food grade Triacetin ${ }^{\circledR}(99.5$ wt. \%) price is approximately $10-15 \%$ higher. Relatively high prices allow the economic viability of even smaller production capacities, ranging from 500 to 1,000 tpy [03].

Triacetin® plant with capacity of 600 tpy consumes annually about $450 \mathrm{t}$ of technical glycerin (or roughly 550-750 tpy of crude bio-glycerin). This is approximately the amount of crude bioglycerin potentially generated by two local FAME plants in operation. Other material inputs of production process are glacial acetic acid and efficient heterogeneous catalyst. In Serbia there is a facility to produce 100,000 tpy of acetic acid within petrochemical complex "MSK" Kikinda.

Production plant consists of two sections: refining of crude bio-glycerin and production of glyc- 
erol-triacetate (reaction system and distillation columns). Around 850,000 EUR. estimates total CAPEX for construction of 600 tpy Triacetin $\AA$ plant.

There are domestic technological recourses for process and plant designing and selecting an optimal catalyst for the process. Establishing of Triacetin $\AA$ production in Serbia, which would be based on use of bio-glycerin refined up to technical grade (98 wt. \%), is estimated as a viable project.

\section{Newly-developed processes}

Drop of glycerin prices, as a consequence of the "boom" in deliveries of bio-glycerin from a dynamically growing biodiesel industry, has opened many opportunities for the valorization of glycerin surpluses through the thermochemical and catalytic conversion towards various chemicals. It is clear that a large number of specialty and fine chemicals can be produced on the basis of reactions involving glycerin, and well-known chemical database Beilstein registers more than 1,500 of such derivatives. Logically, the number of those derivatives that have more serious commercial value is incomparably smaller. Glycerin predominantly substitutes petrochemical propylene in catalytic processes that are commercialized up to now and transferred into industrial practice. Most of these catalytic conversions are at the same time also so called thermochemical processes. The catalytic conversion of bioglycerin into bio-methanol, glycerol ethers or syngas opens up a perspective for enlargement of renewable energy sources, such as bio-dimethyl-ether (Bio-DME), glycerol-tert-butyl-ether (GTBE) or hydrogen (H2). Today, productions of newly-developed organic intermediates participate in global consumption of glycerin with approximately $17 \%$.

Table 3. contains one concise SWOT Analysis, where the crucial strengths, opportunities, weaknesses and threats for each of these glycerinbased chemicals are identified. This analysis included seven newly-developed glycerin-based chemicals that have already been commercialized through the construction of industrial plants or larger pilot plants. Aspecial attention is focused on projects (products) suitable for realization in Serbia. The main conclusion of SWOT Analysis is that at present time for Serbia was only meaningful realization of the project "BioGlycerin-toMPG". Primers for this claim are given below.

\section{Mono propylene glycol (MPG)}

Mono propylene glycol (MPG) is also known as 1,2-propanediol (1,2-PD), and it belongs to the category of medium-tonnage organic chemicals. Industrial grade MPG (min. 95 wt. \%) has many uses, but its main sectors of demand are unsaturated polyester resins (for the production of coatings and glass fiber reinforced plastics) and functional fluids (aircraft deicers, coolants and antifreeze products). Other applications include its uses as plasticizer in production of hydraulic fluid for brake systems and component in syntheses of non-ionic surfactants. MPG is also an excellent extraction solvent. Pharmaceutical grade MPG is at least $99.5 \%$ pure by weight and it finds uses in health-sensitive products such as food, fodder, personal consumer goods, cosmetics, and pharmaceuticals.

Conventionally, the commercial MPG production rote is propylene-based, via propylene-oxide, and therefore it is sensitive to the price of crude oil (or naphtha) and long-term deficiency of propylene. In newly-developed glycerin-based process of MPG production in gaseous phase, glycerin is reacted over a heterogeneous catalyst with hydrogen via a two-step reaction in the same reactor. Glycerin is first dehydrated to acetol, and MPG is then formed by the in-situ hydrogenation of acetol. First step is catalyzed by acid and second-step by metal, so that the commonly used are bi-functional catalysts ( $\mathrm{Cu}-\mathrm{ZnO}-\mathrm{Al} 2 \mathrm{O} 3$ or similar).

The main problem with use of crude bio-glycerin for production of MPG is excessive content of $\mathrm{Na}$ or $\mathrm{K}$ salts. Using of synthesis in gaseous phase opens the possibility of integration with crude bio-glycerin refining as a means of reducing energy consumption and capital expenditures.

Although n-propanol and iso-propanol are being created as by-products of the reaction of glycerin to MPG, they are produced in such small quantities that it is more profitable to simply dispose of them as waste instead of investing in additional purification equipment. The current worldwide market for MPG is around 2 million tpy. Global capacity to produce MPG is around 2.76 million tpy, of which around $30 \%$ is located in Europe [15]. Today, three industrial plants worldwide (US, China and Belgium) use "Glycerin-to-MPG" process, and they together consume about 170,000 tpy of technical glycerin (or roughly 200,000280,000 tpy of crude bio-glycerin) [15]. 
Table 3: Summary of SWOT Analysis for Newly-Developed Glycerin-Based Chemicals

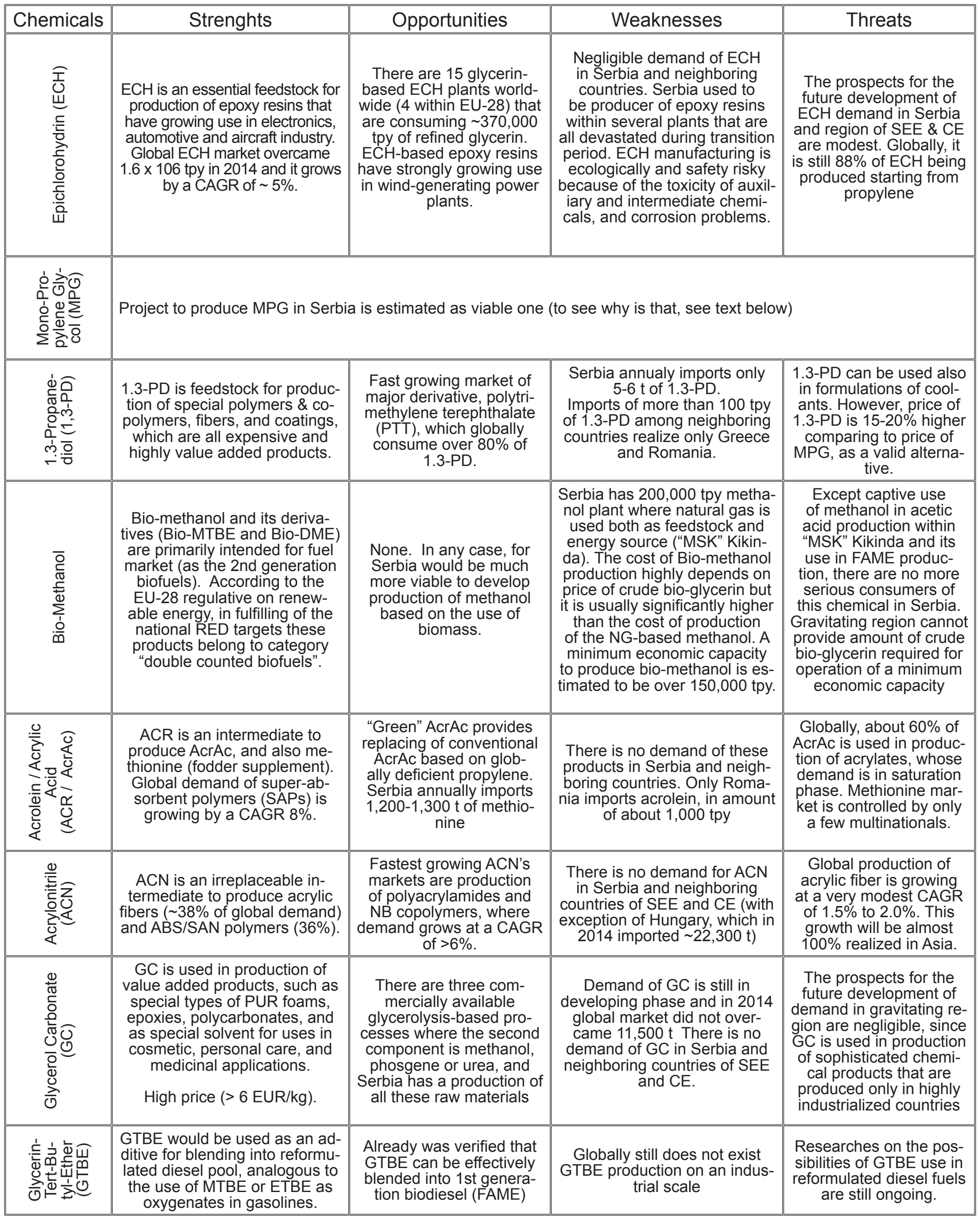


Global demand of MPG grows by a CAGR of about $4 \%$. A significant contribution to the market growth give widespread concerns concerning the toxicity of mono-ethylene glycol (MEG), in the sense that MPG has increasingly replaced MEG in applications where chemical goods come into direct contact with humans and animals. Also, MPG is mandatory to use in refrigeration systems within food industry, where there is a risk of contact with food.

The current prices of MPG industrial grade range from 1,750 to 1,850 EUR per t. Anti-freeze grade costs $8-9 \%$ less whereas the price of pharmaceutical grade is $5-6 \%$ higher [15].

Recent researches on economies of scale suggest that the minimum economic capacity to produce MPG by using "Glycerin-to-MPG" process is about 20,000 tpy. Such a capacity would consume 32,000 tpy to 42,400 tpy of crude bio-glycerin. In some parts of Europe, such as the region of Southeastern Europe, this would might mean the collection of crude bio-glycerin from the several FAME factories [15].

Serbia annually imports (and consumes) over $650 \mathrm{t}$ of MPG, with a growing tendency. However, seven neighboring countries, plus Greece, annually imports over 10,000-11,000 $t$ of MPG. In Central, Eastern and South-Eastern Europe today operates only one 15,000 tpy petroleumbased MPG plant in Romania.

Future development of "glycerin-to-MPG" production in Serbia has marketing sense, but the issue is "economy of scale". To operate above mentioned 20,000 tpy MPG plant an investor could collect around 32,000-42,000 tpy of crude bio-glycerin, what is the quantity that Serbia in the foreseeable future definitely will not have. On the other hand, it is the amount that could still be available through a certain long-term contracting delivery of bio-glycerin from some of the large-scale FAME plants that are operating in the neighboring countries.

\section{CONCLUSION}

Today, when almost total output of glycerin originates from production of biodiesel and oleochemicals, the global availability of glycerin is completely independent of its market demand. In fact, the supply of glycerin depends on dynamics of converting vegetable oil into biodiesel and oleochemicals, which is in turn a function of demand of biofuels and the key sectors of oleochemicals final demand (surfactants, synthetic materials). Thus, demand of glycerin does not have a more significant influence to its prices, which is specificity of only a small number of industrial goods. This explains the importantance of the planned glycerin-based derivative as value added product, whose price is more stable and less fluctuates than the price of glycerin.

New applications in organic chemical synthesis are still far from being absorbed surpluses of glycerin from production of FAMEs and oleochemicals. Even assuming that all industrial plants that currently use the new catalytic processes to convert glycerin into the organics are operating at full capacity, which is not the case, this sector of demand would not overcame the current share within total market of glycerin (crude + refined) of $17-18 \%$. However, there are numerous "BioGlycerin-to-GreenChemicals" projects in phase of construction or designing worldwide (taking into account also the development of new biotechnological conversions of refined glycerin). Hence, there are serious chances that profitable production of "green chemicals" that depart from crude bio-glycerin or refined glycerin will quickly repress the current applications of crude bio-glycerin which are seen as low value added ones (basically incineration) and reduce the surpluses bio-glycerin to a minimum.

Development of the "green-chemistry" based on catalytic conversion of glycerin in Serbia and the region will largely depend on future prices of crude oil and its derivatives. Every more serious price reduction of fossil fuels, as it is the case today, substantially decreases the economic viability of biodiesel industry. And if domestic production of biodiesel does not exist on a more serious industrial scale, then there is no availability of glycerin to be catalytically converted into value-added chemicals.

\section{ACKNOWLEDGMENT}

The project is financially supported by the Ministry of Education, Science and Technological Development Republic of Serbia (Project No. III 45001). 


\section{REFERENCES}

1) BIOFUELS INTERNATIONAL. (2015). SCB Commodity Brokers Global Biofuels Prices. Morden, UK: BIOFUELS INTERNATIONAL.

2) Bogaart, V. (October 20-21, 2009). Glycerin market brief. 7th ICIS World Oleochemicals Conference. Berlin, Germany.

3) Bonet, J., Costa, J., Sire, R., Reneaume, J.M., Plesu, A., Plesu, V., \& Bozga, G. (2009). Revalorization of glycerol: Comestible oil from biodiesel synthesis. Food and Bioproducts Processing(87), 171-178.

4) Ciriminna, R., Della Pina, C., Rossi, M., \& Pagliaro, M. (2014). Special feature: Understanding the glycerol market. European Journal of Lipid Science and Technology(116), 1432-1439.

5) European Commision. (2015). Platts' Spot Oil Products Prices - Rotterdam Quotations Barges - FOB. European Commision.

6) Eurostat. (2016). Primary production of renewable energy by type. http://ec.europa.eu/ eurostat/web/products-datasets/-/ten00081, preuzeto 2016.

7) Fester, G., Wurmseher, M., \& Rammer, C. (2014). Future biofuels production: Price comparison. Biofuels International, 5(8), 6768.

8) Grand View Research Inc. (2014). Glycerol market from biodiesel, fatty acids, fatty alcohols for personal care, alkyd resins, polyether polyols applications, downstreams opportunities and segment forecast to 2020. San Francisco, US: Grang View Research Inc.

9) Gupta, M., \& Kumar, N. (2012). Scope and opportunities of using glycerol as an energy source. Renewable and Sustainable Energy Reviews(16), 4551-4556.

10) Leskovac, S. (June 26, 2014). Review of the current situation at Serbian biofuel market. 2nd Workshop "Implemantation of Directive 2009/28/EC in the Field of Biofuels and Transport in the Republic of Serbia - Transfer of Best Practices" (BIOGOS). Belgrade.

11) Mariano, D., Lucchini, F., \& Coronado, C. (37 November, 2013). Burning of the glycerol as alternative fuel in biodiesel production plants: A case Study. 22nd International congress of mechanical engineering - COBEM. Riberiao Preto, SP, Brazil.
12) Marinković, D., Milanović, P., Popović, Z., Nikolić-Paunić, D., \& Nešić, G. (June 1114, 2012). The future of alternative fuels in transport sector of Serbia. Procedings from CHYMICUS IV - 4th International congres on legal, economic and ecological aspects of the environment management in the chemical, petrochemical and oil industry, (pp. 221229). Tara, Serbia.

13) Marinković, D., Popović, Z., Orlović, A., \& Ristić, M. (2012). Modeling of motor fuels consumption in Serbia, with projection to 2025. Chemical Industry, 3(66), 413-423.

14) OLEOLINE. (2012-2015). Quaterly glycerine market report. Houston, US: HBI Group.

15) Pandia, R. (2014). Markets and potentials for non-polymer derivates of propylene. Shanghai, China: Platts Asia.

16) Souček, I., \& Ocić, O. (2012). Long-term sustainability of bio-components production. Chemical Industry, 2(66), 235-242.

17) Souček, I., Popović, Z., Koprivsek, V., Milićević, M., Leskovac, S., Ocić, O., \& Kubu, M. (2014). Alternative bio-diesel production. Energy/Economy/Ecology, 1-2(16), 49-54.

18) Tan, H., Abdul Aziz, A., \& Aroua , M. (2013). Glycerol production and is applications as a raw materials: A review. Renewable and Sustainable Energy Reviews(27), 118-127.

19) Wu, Y. (2011). Analysis of Triacetine Market. China Chemical Reporter, 22(22), 18.

Paper sent to revision: 18.11.2015.

Paper ready for publication: 26.08.2016. 